\title{
Macrophage dysfunction in Psoriatic Arthritis
}

\author{
Mark H Wenink ${ }^{1 *}$, Kim CM Santegoets ${ }^{1}$, Lenny van Bon ${ }^{1}$, John Butcher ${ }^{2}$, Wim B van den Berg ${ }^{1}$, Piet LCM van Riel ${ }^{1}$, \\ lain B Mclnnes', Timothy RDJ Radstake ${ }^{1}$
}

From 6th European Workshop on Immune-Mediated Inflammatory Diseases

Nice, France. 23-25 November 2011

\section{Introduction}

The pathogenesis of Psoriatic Arthritis (PsA) remains poorly understood. The underlying chronic inflammatory immune response is thought to be triggered by unknown environmental factors and might arise due to an impaired (innate) immune function by Dendritic Cells (DCs) [1]. Anti-inflammatory $\mathrm{CD} 163^{+}$type 2 macrophages $(\mathrm{mf}-2)$ are thought to have important functions in restoring immune homeostasis during an inflammatory response. Mf- 2 are present in PsA synovium at high numbers. Why the immune response in PsA goes awry, despite the presence of these $\mathrm{mf}-2$, is still largely undetermined.

\begin{abstract}
Aim
We aimed to determine whether $\mathrm{mf}-2$ from PsA patients have an aberrant phenotype thereby contributing to the chronic inflammation occurring in PsA.
\end{abstract}

\section{Patients and methods}

Mf-2 from 12 healthy controls and 12 PsA patients were analysed for their expression of various cell surface receptors as well as their cytokine production when exposed to a range of bacteria and single Toll-Like receptor (TLR) ligands. Differences between healthy control and PsA mf-2 involved in suppressing DC function were determined by stimulation assays and mixed leukocyte reactions.

\section{Results}

CCR5 was significantly increased on $\mathrm{mf}-2$ from PsA patients compared to healthy controls while the expression of CCR1, TLR2, TLR4 and CD14 was unaltered. Further research revealed that PsA mf- 2 secreted more
IL-6 upon incubation with heat-killed Escherichia coli and $E$. coli lipopolysaccharide and were less efficient in phagocytosing E. coli. The production of TNFa and IL10 was equivalent between the groups. The production of cytokines was equal between PsA and healthy control mf-2 upon the stimulation with $M$. tuberculosis, P. gingivalis or TLR 2 or TLR7/8 ligands. In addition, PsA mf-2 were less capable of suppressing cytokine production by DCs compared to mf-2 from healthy controls. Further reflecting their disordered function was the finding that in T cell assays PsA mf- 2 are less capable of suppressing DC induced IL-17 and TNF $\alpha$ production by T cells.

\section{Conclusion}

Anti-inflammatory $\mathrm{CD}_{163^{+}} \mathrm{mf}-2$ from PsA patients have a clearly aberrant phenotype. This implicates $\mathrm{mf}-2$ as an important defective cell type in PsA facilitating chronic inflammation instead of exerting their normal function of restoring immune homeostasis.

\section{Author details}

${ }^{1}$ Dept. of Rheumatology, Nijmegen Center of Infection, Inflammation and Immunity (N4i) and Nijmegen Centre for Molecular Life Science (NCMLS), Radboud University Nijmegen Medical Centre, Nijmegen, The Netherlands. 2Division of Immunology, Infection and Inflammation, Glasgow Biomedical Research Centre, University of Glasgow, Glasgow, Scotland, UK.

Published: 23 November 2011

Reference

1. Wenink MH, Santegoets KC, Butcher J, van Bon L, Lamers-Karnebeek FG, van den Berg WB, et al: Impaired dendritic cell proinflammatory cytokine production in psoriatic arthritis. Arthritis Rheum 2011.

doi:10.1186/1479-5876-9-S2-P59

Cite this article as: Wenink et al:: Macrophage dysfunction in Psoriatic Arthritis. Journal of Translational Medicine 2011 9(Suppl 2):P59. 